\title{
Docomomo Ibérico propone incorporar 140 nuevas obras al Plan Nacional de Protección del Patrimonio Cultural del siglo XX
}

\begin{abstract}
La Fundación DOCOMOMO Ibérico ha llevado a cabo estos últimos cuatro años una ampliación temporal de los registros que elabora sobre el Movimiento Moderno en España y Portugal, con el fin de abordar el periodo comprendido entre 1965 y 1975. El pasado mes de abril en unas jornadas de trabajo, de los 350 nuevos edificios registrados, se seleccionaron 140, como los más significativos, para su incorporación al Plan Nacional de Protección del Patrimonio Cultural del siglo XX, coordinado por el Instituto del Patrimonio Cultural de España, para gozar del máximo nivel de protección.
\end{abstract}

Susana Landrove Bossut | Fundación DOCOMOMO Ibérico

URL de la contribución <http://www.iaph.es/revistaph/index.php/revistaph/article/view/4405>

La primera labor a la que se enfrentó DOCOMOMO Ibérico cuando inició su actividad, en 1993, fue la de determinar qué podía significar el Movimiento Moderno, en el ámbito de España y Portugal, y cuál había sido la extensión de su implantación. Para ello, se delimitó un periodo de estudio que quedó comprendido entre 1925 y 1965 . La primera fecha, por ser el momento de concepción de las primeras obras que podían inscribirse sin objeciones en el lenguaje de la modernidad, y 1965, por estimar que ese año, además de garantizar el necesario distanciamiento de los hechos objeto de estudio, escenificaba la puesta en cuestión de los planteamientos del Movimiento Moderno, con la publicación en España de textos como los de Aldo Rossi, La arquitectura de la ciudad, o de Robert Venturi, Complejidad y contradicción en la arquitectura. Esa primera labor de aproximación a la arquitectura del Movimiento Moderno quedó reflejada en el registro que se publicó, en 1996, bajo el título La arquitectura del movimiento moderno. Registro DOCOMOMO Ibérico, 1925-1965.

Tras este primer trabajo, se realizaron tres registros temáticos pormenorizados (sobre la industria, la vivienda y los equipamientos), que pusieron en evidencia cómo la incidencia de la arquitectura moderna en los territorios ibéricos fue mucho mayor de lo que la literatura existente sobre el tema había reflejado, extendiéndose más allá de los polos que tradicionalmente se habían considerado como el núcleo geográ- fico de la arquitectura moderna. Gracias a estos trabajos, se documentaron 1.736 edificios y conjuntos que quedaron recogidos en cuatro publicaciones.

Pero si el trabajo de documentación se podía dar, en cierta medida, por concluido, no era así con la protección de los edificios recogidos en los registros. La gran mayoría carecía de protección y sus valores ignorados por la sociedad. En ese sentido, la incorporación, en 2014, al Plan Nacional de Protección del Patrimonio Cultural del siglo XX, coordinado por el Instituto del Patrimonio Cultural de España, de los 256 elementos que una comisión de expertos, externa a DOCOMOMO, consideró de mayor valor, supuso un importante respaldo, pues quedaban equiparados con otros patrimonios, como las catedrales, los monasterios y abadías, o la industria, que el Ministerio de Educación, Cultura y Deportes había considerado también merecedores de Planes Nacionales de Protección equivalentes al que nos ocupa.

Si los trabajos de registro de DOCOMOMO se habían enmarcado hasta entonces en el periodo comprendido entre 1925 y 1965, con el paso del tiempo pareció justificable abarcar un periodo más amplio. Efectivamente, la realización de los diferentes registros puso de manifiesto cómo muchas obras que quedaron excluidas de los registros DOCOMOMO por superar la fecha de 1965 eran perfectamente adscribibles a la modernidad arquitectónica. Por otra parte, con el paso de los años, había variado la percepción de la modernidad, perdiendo peso 

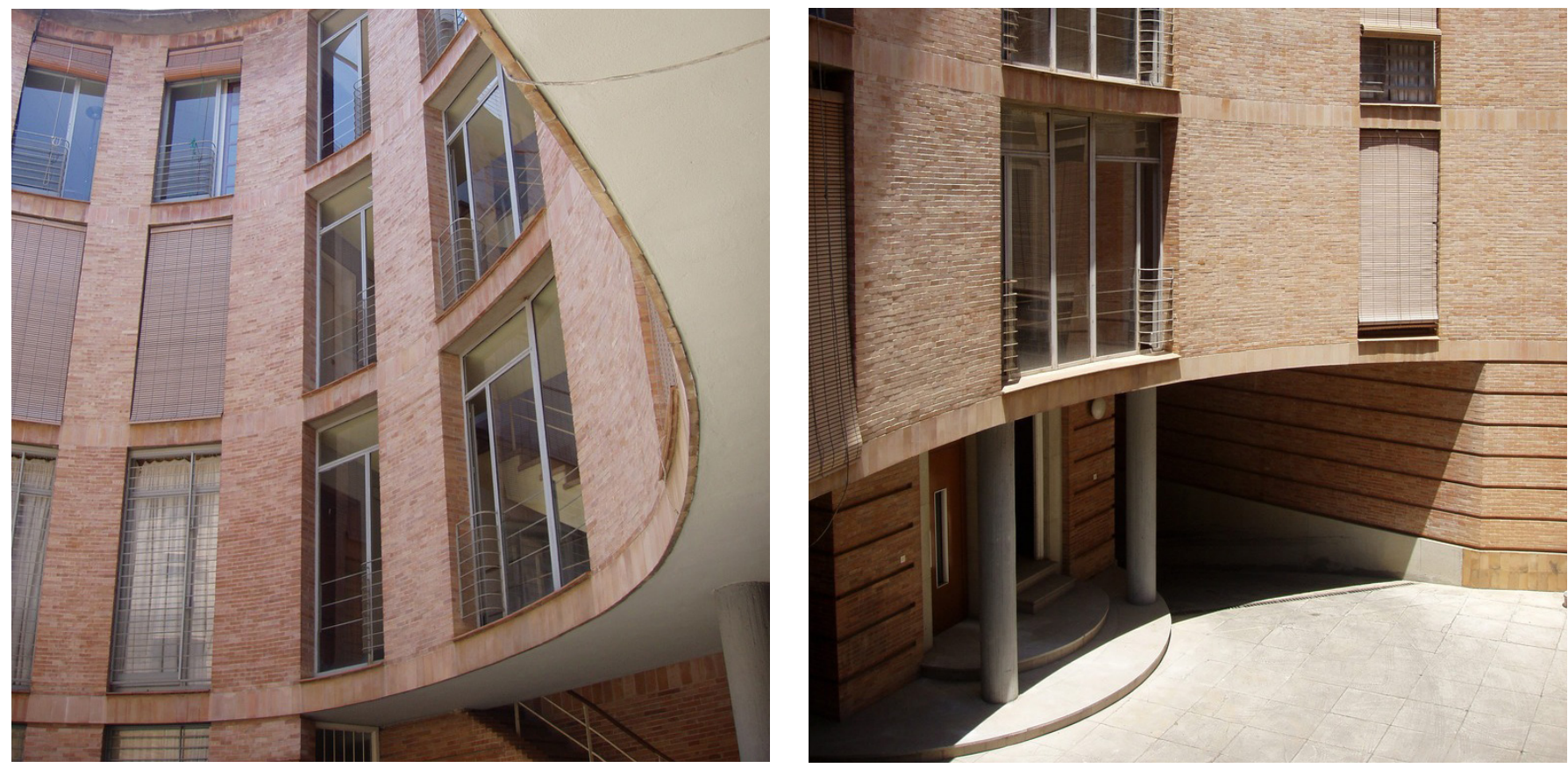

Viviendas en calle Doña María Coronel, Sevilla (Cruz y Ortiz, 1974-1976). Vistas del interior del patio. Una de las obras seleccionadas por DoCoMoMo Ibérico para el Registro 1965-1975 (Tipo A) | fotos Fondo Gráfico IAPH (Ignacio Capilla Roncero, Amadeo Ramos Carranza, Ignacio Sánchez-Cid Endériz)

algunos argumentos y cobrando valor otros. La ortodoxia parecía haber perdido peso frente a los márgenes y transgresiones que se revelan ahora como un valor propio de las arquitecturas del ámbito ibérico. Fruto de estas consideraciones, en 2014 , se planteó la necesidad ampliar la cronología de estudio de DOCOMOMO Ibérico hasta el final de los regímenes autoritarios de España y Portugal, fijándose el nuevo periodo de estudio entre 1965 y 1975 . Como no podía ser de otro modo, la adopción de esa cronología ha hecho emerger nuevas dudas y plantear exclusiones que, posiblemente, puedan ser revisadas más adelante. Este nuevo trabajo ha supuesto el estudio, por parte de la comisión técnica de DOCOMOMO Ibérico, de 1.110 edificios, de los que se han incorporado a los registros DOCOMOMO 350, correspondientes al periodo comprendido entre 1965 y 1975. Y, de nuevo, se ha hecho necesario destacar una selección de aquellos susceptibles de incorporarse al Plan Nacional, completando una franja cronológica más del ambicioso proyecto que auspicia el Instituto de Patrimonio Cultural de España con el fin de instar a la conservación del patrimonio arquitectónico del siglo $\mathrm{XX}$. Con ese objetivo, una nueva comisión de expertos se reunió a mediados de abril en sesiones de trabajo y en unas jornadas que presentaron al público algunas especificidades de las arquitecturas de ese periodo, desde diferentes perspectivas, planteando algunos de los aspectos que han articulado la selección de obras a incorporar al Plan Nacional. Son estos aspectos relativos a la contribución resistente de la forma, a los personajes de ese periodo que adquieren protagonismo desde una visión contemporánea, a los vínculos entre tradición y modernidad, y a las continuidades de las cronologías de estudio pero también a las contradicciones y los límites que de ésta afloran, así como los aspectos vinculados al patrimonio y la conservación de la vivienda social o las arquitecturas que se perdieron.

Fruto de las sesiones de trabajo, hay una propuesta de unas 140 obras que se han considerado que, junto a las 256 ya incorporadas al Plan Nacional, deberían gozar del máximo nivel de protección y cuyos valores hemos de ser capaces de transmitir a la sociedad así como a ayuntamientos y autonomías que deberían tomar este acerbo como orientación y establecer las estrategias adecuadas para garantizar su permanencia. 